\title{
Umidade de equilíbrio da madeira de angelim vermelho (Dinizia excelsa Ducke), guariúba (Clarisia racemosa Ruiz \& Pav.) e tauarí vermelho (Cariniana micrantha Ducke) em diferentes condições de temperatura e umidade relativa.
}

\author{
Edy Eime Pereira BARAÚNA ${ }^{1}$, Valmir Souza DE OLIVEIRA²
}

\begin{abstract}
RESUMO
A umidade de equilíbrio deve ser determinada para o local onde a madeira será empregada. Isto pode ser feito através da determinação da umidade das amostras de madeira expostas às condiçôes ambientais de temperatura e umidade relativa em ensaios de campo, de laboratório equipado com câmara de climatização ou estimativas por meio de modelos matemáticos. Neste trabalho foi determinada a umidade de equilíbrio da madeira - UEM do angelim vermelho (Dinizia excelsa Ducke), guariúba (Clarisia racemosa Ruiz \& Pav.) e tauarí vermelho (Cariniana micrantha Ducke ), em duas condições de temperatura e três de umidade relativa em câmara de climatização. Encontrou-se diferenças entre a umidade de equilíbrio estimada pela equação de Simpson (1971) e o valor real determinado em câmara climática. Na simulação de ensaio a $25^{\circ} \mathrm{C}$ de temperatura e umidade relativa de $40 \%$ a UEM ficou em média 26,6\% superior ao valor estimado pela equação de Simpson (1971), constituindo-se na maior variaçãa. A menor variação foi de $2,1 \%$ registrada na espécie angelim vermelho na condição de $35^{\circ} \mathrm{C}$ e $80 \%$ de umidade relativa. A equação de Simpson, no geral, tendeu a superestimar os valores de UEM nas três espécies.
\end{abstract}

PALAVRaS-Chave: Madeira, Umidade de equilíbrio, Câmara de climatização, Equação de Simpson, Secagem da madeira.

\section{Wood equilibrium moisture content of angelim vermelho (Dinizia excelsa Ducke ), guariúba (Clarisia racemosa Ruiz \& Pav.) and tauarí vermelho (Cariniana micrantha Ducke) at different conditions of temperature and relative humidity}

\begin{abstract}
The equilibrium moisture content (EMC) of wood must be determined according to the place where wood is to be used. Moisture content can be determined from wood samples exposed to environmental conditions of temperature and relative humidity in the field, a laboratory equipped with environmental chambers, or by mathematical model calculations. The EMCs of angelim vermelho (Dinizia excelsa Ducke), guariúba (Clarisia racemosa) and tauarí vermelho (Cariniana micrantha Ducke) were determined in a environmental chamber at two temperatures and three relative humidity conditions. Differences were found between the EMC estimated by the Simpson's equation (1971) and the real value determined in environmental chamber. Large discrepancies, averaging $26,6 \%$, were found at $25^{\circ} \mathrm{C}$ and $40 \%$ relative humidity. The minimum discrepancies, averaging $2,1 \%$, were determined on angelim vermelho at $35^{\circ} \mathrm{C}$ and $80 \%$ relative humidity. In general, the Simpson's equation overestimated the EMC values for the three species.
\end{abstract}

KEYWORDS: Wood, Equilibrium moisture content, Environmental chamber, Equation of Simpson, Wood drying.

1 Professor da Universidade Federal de Tocantins. Caixa Postal 66, CEP 77402-97 - Campos de Gurupi/TO. E-mail: ebarauna@posgrad.ufla.br

2 In memorian 


\section{INTRODUÇÃO}

Quando serrada a madeira possui um determinado conteúdo de água, e sendo constituída por células porosas, a evaporação acontecerá até que seja atingido um equilíbrio entre a atmosfera envolvente e a madeira em uso (Wengert, 2005).

O teor de umidade da madeira quando em serviço sofre variaçōes em função da umidade relativa e da temperatura ambiental em que se encontra a peça (Skaar, 1988). Fixandose essas variáveis, o teor de água da madeira ajusta-se a um valor de equilíbrio. Esse fenômeno torna possível a estimativa da umidade da madeira utilizada em diferentes condiçôes ambientais.

A umidade de equilíbrio deve ser determinada para o local onde a madeira será empregada, o que pode ser conseguido com o uso de amostras de madeira expostas às condiçôes ambientais de temperatura e umidade relativa, podendose utilizar ensaios de campo, secador artificial, câmara de climatização ou estimativas por meio de equaçóes.

Dentre as diversas teorias da sorção da água na madeira, a mais empregada é a de Hailwood e Harrobin e vários autores vem empregando-a para estimar a umidade de equilíbrio em várias cidades brasileiras (Galvão, 1975 e 1981; Lima \& Mendes, 1995; Lima et al. 2000 e Martins et al. 2003).

Entretanto, eventuais diferenças entre valores de umidade de equilíbrio estimados por equaçóes e os valores reais devem ser determinadas e que, para isso, necessita-se conhecer a umidade de equilíbrio real para as principais madeiras consumidas nas cidades brasileiras, e mais especificamente no município de Manaus - AM.

$\mathrm{Na}$ floresta amazônica, existem inúmeras espécies com potencial para abastecer a demanda de madeira para o mercado local, nacional e internacional. Entretanto, poucos estudos são realizados com essas essências, para avaliar o comportamento frente ao desdobro, uso, trabalhabilidade e estimativa do valor real da umidade de equilíbrio.

O objetivo deste estudo foi determinar a umidade de equilíbrio para as madeiras de angelim vermelho (Dinizia excelsa Ducke), guariúba (Clarisia racemosa Ruiz \& Pav.) e o tauarí vermelho (Cariniana micrantha Ducke ) em duas condiçōes de temperatura e três condiçōes de umidade relativa em câmara de climatização, além de compará-las com aquelas estimadas pela equação de Simpson (1971).

\section{MATERIAIS E MÉTODOS}

\section{OBTENÇÃO DA MADEIRA}

A madeira de angelim vermelho (Dinizia excelsa Ducke), guariúba (Clarisia racemosa Ruiz \& Pav.) e tauarí vermelho
(Cariniana micrantha Ducke) utilizada neste experimento foi fornecida por uma empresa madeireira certificada sediada no município de Itacoatiara, estado do Amazonas, sendo uniformizada em tábuas de 2,5 × 19,0 × 200,0 cm, com quatro tábuas por espécie. Essas espécies foram identificadas através de observação macroscópica da anatomia da madeira no Instituto Nacional de Pesquisa da Amazônia - INPA.

\section{PREPARO DO CORPO-DE-PROVA}

Para esta pesquisa foram utilizados corpos-de-prova com dimensōes de 2,5 cm de espessura x 5,0 cm de largura x $10 \mathrm{~cm}$ de comprimento, sendo quatro repetiçôes por espécie (Figura 1). Estes foram colocados em uma câmara de climatização para simulação experimental da umidade de equilíbrio.

Foram simuladas duas condiçōes de temperatura e três de umidade relativa (Tabela 1), as quais englobam as médias de temperatura e umidades relativas típicas como também as médias extremas da cidade de Manaus - AM.

Periodicamente as amostras eram retiradas da câmara de climatização, acondicionadas em plásticos para evitar trocas de umidade com o ambiente e foram pesadas em balança eletrônica digital, com precisão de $\pm 0,01 \mathrm{~g}$. Foram realizadas pesagens consecutivas, considerando-se o equilíbrio quando a diferença de peso era $\leq \mathrm{a} 0,02 \mathrm{~g}$. Nesta condição, a amostra tinha seu peso de equilíbrio registrado e dava-se seqüência a outra condição.

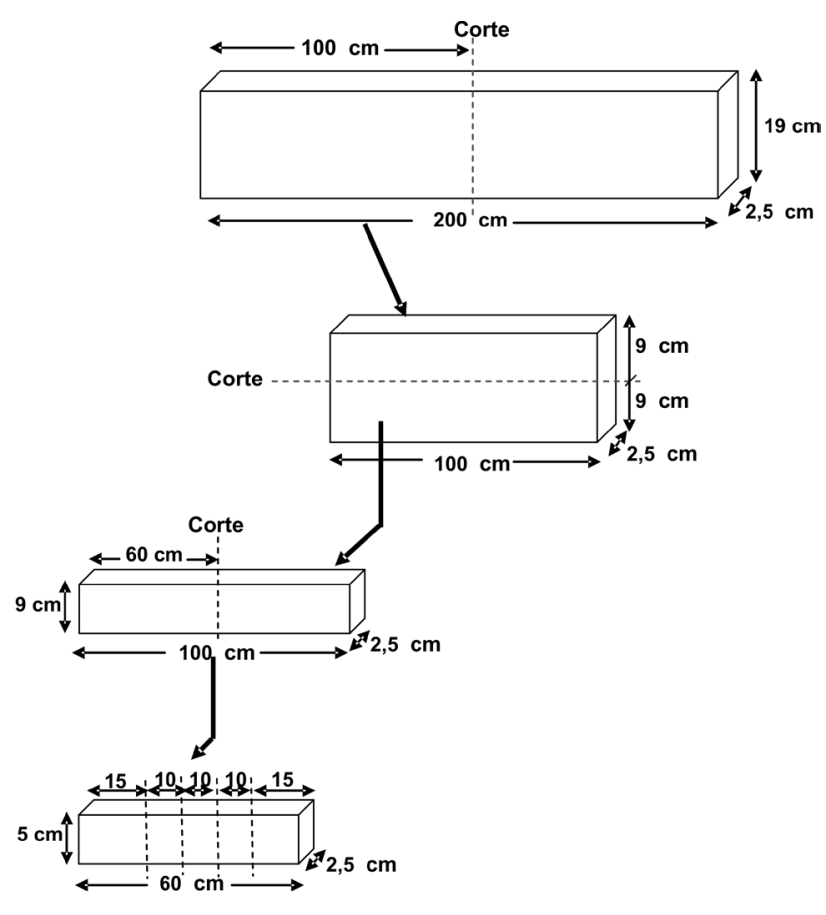

Figura 1 - Esquema da amostragem para o experimento. 
Tabela 1 - Seqüência de acondicionamento das amostras na câmara climática em diferentes umidades relativa, temperatura e correspondentes umidades de equilíbrio.

\begin{tabular}{llll}
\hline Condição & Temperatura $\left({ }^{\circ} \mathrm{C}\right)$ & Umidade relativa $(\%)$ & $\mathrm{UE}^{\star}(\%)$ \\
\hline 1 & 35 & 80 & 15,46 \\
2 & 35 & 60 & 10,39 \\
3 & 35 & 40 & 7,14 \\
4 & 25 & 80 & 16,01 \\
5 & 25 & 60 & 10,87 \\
6 & 25 & 40 & 7,49 \\
\hline
\end{tabular}

* Umidade de equilíbrio estimada pela equação de Simpson (1971).

Ao final de todas as condições experimentais para o cálculo da umidade em cada condição pré-estabelecida foi determinada a massa seca das amostras através da secagem em estufa a uma temperatura de $103 \pm 2{ }^{\circ} \mathrm{C}$ por um período de 48 horas. Também, foram obtidos os volumes secos das amostras para determinação da densidade seca. Os volumes foram determinados por medição das dimensões das amostras usando um paquímetro digital.

A estimativa da umidade de equilíbrio da madeira - UEM foi obtida pelo uso da equação de Simpson (1971) sendo:

$$
\mathrm{UEM}=\left[\frac{K_{1} K_{2} h}{1+K_{1} K_{2} h}+\frac{K_{2} h}{1-K_{2} h}\right] \frac{1800}{W}
$$

Em que:

UEM = umidade de equilíbrio da madeira (\%)

$$
\begin{aligned}
& \mathrm{K}_{1}=4,737+0,04773 \cdot \mathrm{T}-0,00050123 \cdot \mathrm{T}^{2} \\
& \mathrm{~K}_{2}=0,705941+0,001698 \cdot \mathrm{T}-0,000005553 \cdot \mathrm{T}^{2} \\
& \mathrm{~W}=223,384+06942 \cdot \mathrm{T}+0,0185324 \cdot \mathrm{T}^{2} \\
& \mathrm{~T}=\text { temperatura em graus Celsius } \\
& \mathrm{h}=\text { pressão relativa de vapor d'água. }
\end{aligned}
$$

\section{RESULTADOS E DISCUSSÃO}

A Tabela 2 indica a densidade seca (DS), as umidades de equilíbrio e os valores estimados pela equação de Simpson (1971) para as três espécies ensaiadas na câmara climática nas temperaturas de $35^{\circ} \mathrm{C}$ e $25^{\circ} \mathrm{C}$ e umidades relativas de $80 \%, 60 \%$ e $40 \%$.

Analisando os resultados individuais por espécie na Tabela 2, foi observado, como era esperado, que, a medida que elevase a umidade relativa em temperatura constante de $25^{\circ} \mathrm{C}$ ou $35^{\circ} \mathrm{C}$, a UEM aumenta. $\mathrm{O}$ tauari vermelho apresentou valores de umidade de equilíbrio muito superiores aos estimados pela equação de Simpson (1971), exceto para a condição de $25^{\circ} \mathrm{C}$ de temperatura e umidade relativa de $80 \%$. A maior diferença entre a umidade de equilíbrio estimada pela equação de Simpson e o valor real ocorreu para o tauari na condição de $25^{\circ} \mathrm{C}$ de temperatura e umidade relativa de $40 \%$, a qual ficou em média $26,6 \%$ superior ao valor encontrado pela equação de Simpson (1971). Enquanto a menor variação $(2,1 \%)$ ocorreu para o angelim vermelho na condição de $35^{\circ} \mathrm{C}$ de temperatura e $80 \%$ de umidade relativa.

A Figura 2 apresenta na forma de histograma os dados obtidos na Tabela 2 e os estimados pela equação de Simpson (1971).

O angelim vermelho e a guariúba obtiveram, com exceção à $25^{\circ} \mathrm{C}$ e $80 \%$ U.R., valores reais de UEM menores quando comparados com os dados estimados pela equação de Simpson. Para elevada umidade relativa $(80 \%)$, independentemente da temperatura e as demais umidades relativas (60\% e $40 \%)$, os valores de UE da madeira encontrados na câmara de climatização estiveram maiores do que os dados estimados (Figura 2).

Segundo Jankowsky (1979) existe uma correlação do

\begin{tabular}{|c|c|c|c|c|c|c|c|}
\hline \multirow[b]{3}{*}{ Espécie } & \multirow[b]{3}{*}{$\mathrm{DS}\left(\mathrm{g} / \mathrm{cm}^{3}\right)$} & \multicolumn{6}{|c|}{ Umidade de equilíbrio (\%) } \\
\hline & & \multicolumn{3}{|c|}{$35^{\circ} \mathrm{C}$} & \multicolumn{3}{|c|}{$25^{\circ} \mathrm{C}$} \\
\hline & & $80 \%$ U.R. & $60 \%$ U.R. & $40 \%$ U.R. & $80 \%$ U.R. & $60 \%$ U.R. & $40 \%$ U.R. \\
\hline Angelim vermelho & 1,010 & $\begin{array}{c}15,13 \\
(2,1 \%)^{\star}\end{array}$ & $\begin{array}{c}12,30 \\
(18,3 \%)\end{array}$ & $\begin{array}{c}8,4 \\
(18,2 \%)\end{array}$ & $\begin{array}{c}13,71 \\
(14,4 \%)\end{array}$ & $\begin{array}{c}12,19 \\
(12,2 \%)\end{array}$ & $\begin{array}{c}9,43 \\
(25,9 \%)\end{array}$ \\
\hline Guariúba & 0,662 & $\begin{array}{l}14,09 \\
(8,9 \%)\end{array}$ & $\begin{array}{c}11,09 \\
(6,7 \%)\end{array}$ & $\begin{array}{c}7,7 \\
(8,5 \%)\end{array}$ & $\begin{array}{c}13,47 \\
(15,9 \%)\end{array}$ & $\begin{array}{l}11,49 \\
(5,7 \%)\end{array}$ & $\begin{array}{c}8,94 \\
(19,4 \%)\end{array}$ \\
\hline Tauari & 0,702 & $\begin{array}{l}16,53 \\
(6,9 \%)\end{array}$ & $\begin{array}{c}12,97 \\
(24,8 \%)\end{array}$ & $\begin{array}{c}8,40 \\
(17,7 \%)\end{array}$ & $\begin{array}{c}15,42 \\
(3,7 \%)\end{array}$ & $\begin{array}{c}12,78 \\
(17,6 \%)\end{array}$ & $\begin{array}{c}9,48 \\
(26,6 \%)\end{array}$ \\
\hline Média & & $\begin{array}{l}15,25 \\
(1,4 \%)\end{array}$ & $\begin{array}{c}12,12 \\
(16,6 \%)\end{array}$ & $\begin{array}{c}8,20 \\
(14,7 \%)\end{array}$ & $\begin{array}{c}14,20 \\
(11,3 \%)\end{array}$ & $\begin{array}{c}12,15 \\
(11,8 \%)\end{array}$ & $\begin{array}{c}9,28 \\
(23,9 \%)\end{array}$ \\
\hline E. Simpson (1971) & & 15,46 & 10,39 & 7,14 & 16,01 & 10,87 & 7,49 \\
\hline
\end{tabular}
teor de extrativos e a densidade na madeira, ou seja, quanto maior esta correlação, mais elevado o seu efeito na umidade de equilíbrio, e conseqüentemente um decréscimo na UEM.

Tabela 2 - Valores médios (\%) para a UEM das espécies de Dinizia excelsa, Clarisia racemosa e Cariniana micrantha obtida na câmara de ensaios climáticos.

* Valores entre parênteses significam a diferença em \% dos valores obtidos na câmara climática aqueles estimados pela equação de Simpson (1971). 


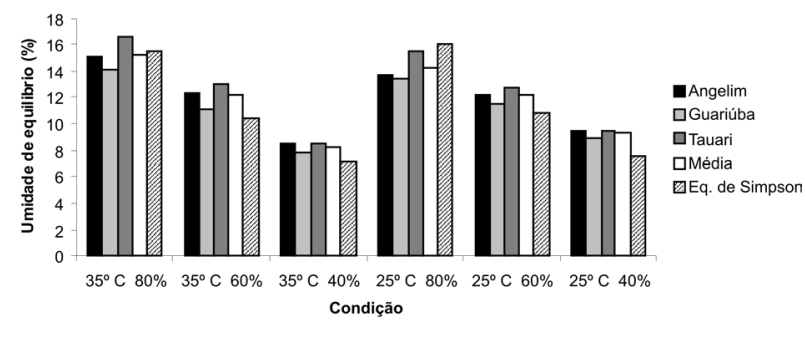

Figura 2 - Valores da umidade de equilíbrio obtidos na câmara de ensaios climáticos e estimados pela equação de Simpson (1971).

Wangaard \& Granados (1967) ao analisarem o efeito dos extrativos na sorção do vapor de água em nove espécies de madeira oriundas de floresta tropical observaram que os extrativos contribuem para a higroscopicidade em escalas mais baixas de umidades relativas e que o efeito dos extrativos depende muito da higroscopicidade do próprio extrativo. Possivelmente o alto conteúdo de extrativos presentes na madeira de tauarí influenciou na UEM desta ocasionando um aumento significativo. Entretanto, mas trabalhos devem ser realizados para confirmar essa higroscopicidade.

As três espécies utilizadas neste trabalho são descritas por Loureiro e Silva, (1968), Mainieri \& Chimelo, (1989) e Rizzini, (2000) como sendo de madeira moderadamente pesada (Cariniana micrantha) a muito pesada (Dinizia excelsa).

Trugilho et al. (2000) ao estimar a umidade de equilíbrio para 27 espécies comerciais de madeiras com uso de câmara de climatização observou que a equação de Simpson (1971) provocou grandes erros de estimativas para todas as espécies avaliadas, sendo que superestima a UEM das madeiras de alta densidade e subestima as de baixa densidade. Neste trabalho cerca de $80 \%$ dos valores de UEM encontrados na câmara climática foram maiores que na equação de Simpson (1971).

Apesar da equação de Simpson (1971) ser muito empregada para estimar a umidade de equilíbrio, estes resultados indicam uma determinada cautela com o seu uso, uma vez que podem ocorrer grandes erros, dependendo da condição estabelecida e da espécie de madeira.

\section{CONCLUSÃO}

Com base nos resultados encontrados no presente trabalho conclui-se que:

Nas três espécies estudadas para a condição de temperatura de $25^{\circ} \mathrm{C}$ e umidade relativa de $80 \%$, a umidade de equilíbrio estimada pela equação de Simpson (1971) tende a ser maior do que os valores reais de UEM;
O tauarí em ambientes com temperatura de $35^{\circ} \mathrm{C}$ e umidades relativas de $60 \%$ e $80 \%$ possui umidades de equilíbrio superiores quando comparado com valores de estimativa da equação de Simpson (1971);

Para elevadas umidades relativas $(80 \%)$, independente da temperatura, o angelim vermelho e a guariúba tendem a obterem UEM maiores que os estimados pelo modelo matemático de Simpson (1971);

A equação de Simpson (1971) gera valores de UEM insatisfatórios para madeiras em Manaus oriundas de florestas tropicais, onde as essências florestais apresentam muitas variaçōes até mesmo entre indivíduos da mesma espécie. $\mathrm{O}$ ideal é ajustar esta estimativa por espécie.

\section{BIBLIOGRAFIA CITADA}

Galvão, A.P.M. 1975. Estimativas da umidade de equilíbrio da madeira em diferentes cidades do Brasil. Instituto de Pesquisa Florestal-IPEF, 11:53-65.

Galvão, A.P.M. 1981. A umidade de equilíbrio e a secagem da madeira em Brasília, Boletim de Pesquisa Florestal, 3: 1-7.

Jankowsky, I.P. 1979. Influência da densidade básica e do teor de extrativos na umidade de equilíbrio da madeira. Dissertação de mestrado, Escola Superior de Agricultura Luiz de Queiroz ESALQ/USP, Piracicaba, São Paulo, 94pp.

Lima, J.T.; Mendes, L.M. 1995. Estimativa da umidade de equilíbrio para madeiras em Lavras-MG, Comunicação, Ciência e Prática, 19(1): 120-121.

Lima, J.T.; Trugilho, P.F.; Silva, J.R.M.; Cruz, C.R. 2000. Comparação entre métodos para estimação da umidade de equilíbrio em madeiras. In: Encontro Brasileiro em Madeiras e em Estruturas de Madeira (VII EBRAMEM), São Carlos-SP, RESUMOS, p. 12-12.

Loureiro, A.A.; Silva, M.F. 1968. Catálogo das Madeiras da Amazônia. Instituto Nacional de Pesquisas da Amazônia - INPA, vol.2, p.185- 187 .

Mainieri, C.; Chimelo, J.P. 1989. Fichas de características das madeiras brasileiras, Instituto de Pesquisas Tecnológicas do Estado de São Paulo, 2a. edição, 428pp.

Martins, V.A.; Alves, M.V.S.; Silva, J.F.; Rebello, E.R.G.; Pinho, G.S.C. 2003. Umidade de equilíbrio e risco de apodrecimento da madeira em condições de serviço no Brasil. Brasil Florestal, 76: $29-34$.

Rizzini, C.T. 2000. Árvores e madeiras úteis do Brasil: Manual de dendrologia brasileira, Rio de Janeiro, Edgard Blucher, 296pp.

Simpson, W.T. 1971. Equilibrium moisture content prediction for wood, Forest Products Journal, 21(5): 48-49.

Skaar, C. 1988. Wood water relations. Department of Forest Products, Virginia Polytechnic Institute and State University, Springer-Verlag Berlin Heidelberg, New York - USA, 283pp. 
AMAZONICA

Trugilho, P.F.; Lima, J.T.; Mori, F.A.; Silva, J.R.M.; Mendes, L.M.; Pinto, L.V.A. 2000. Umidade de equilíbrio da madeira para diferentes condiçôes de temperatura e umidade relativa em câmara de climatização, In: Encontro Brasileiro em Madeiras e em Estruturas de Madeira (VII EBRAMEM), 2000, São CarlosSP, - RESUMOS, p. 41-41.

Wangaard, F.F.; Granados, L.A. 1967. The effect of extractives on water-vapor sorption by wood. Wood Science and Tecnology, 1: 253-277.

Wengert, G. 2005. Measuring moisture content. Cabinet Maker, 19(2): 26-27.

Recebido em 17/09/2007

Aceito em 19/07/2008 
